frustration adversely impacted aspects of clinical reasoning. Students struggled when witnessing what they considered 'bad' behaviour as it contradicted their previously held ideals of how physicians should act.

It seems we teach students to try to internalise emotion yet that it is acceptable to let it negatively impact patient care. To combat this, students sought greater emotional transparency from physicians as well as advice on self-management strategies. Clinicians recognised the benefits of being candid but were afraid of being so. Contributing to this is the culture in medicine being one that mistrusts emotion. Further, both groups desired a formal curriculum addressing emotion in clinical reasoning thus suggesting one is needed.

\section{ACUTE MAGNETIC RESONANCE IMAGING FOR MILD TRAUMATIC BRAIN INJURY}

${ }^{1}$ Sophie Richter, ${ }^{2}$ Stefan Winzeck, ${ }^{1}$ Evgenios Kornaropoulos, ${ }^{3}$ Tilak Das, ${ }^{1}$ Guy B Williams, ${ }^{1}$ Marta M Correia, 'David Menon, ${ }^{1}$ Virginia Newcombe. ${ }^{1}$ Cambridge University; ${ }^{2}$ Cambridge University/Imperial College London; ${ }^{3}$ Addenbrooke's Hospital, Cambridge

10.1136/emj-2020-rcemabstracts. 29

Aims/Objectives/Background Mild traumatic brain injury (mTBI) accounts for one million emergency department attendances in the UK every year. Whilst $30-50 \%$ of patients suffer from persistent symptoms, unselected follow up would overwhelm the health care system. Magnetic resonance imaging (MRI), may help to stratify patients for clinical follow up and interventional trials. We therefore aimed to identify:

1. Neuroanatomical features of concussion on MRI and

2. the optimal timing for magnetic resonance imaging $(<72 \mathrm{~h}$ or 2-3 weeks after injury).

This is the largest study to date using serial scanning acutely in patients with mTBI.

Methods/Design Data originated from two prospective cohorts: the Collaborative European NeuroTrauma Effectiveness Research in Traumatic Brain Injury (CENTER-TBI) study (2014-2017) and a local cohort (2012-2013). Eligible patients presented to hospital within 24h of a mTBI (Glasgow Coma Score 13-15), satisfied local criteria for computed tomography scanning and received two MRIs: one within $72 \mathrm{~h}$ (MR1) and one 2-3 weeks after injury (MR2). In addition, 104 controls were enrolled. Volumes and diffusion parameters for brain regions of interest were extracted via automated pipelines. Symptoms were measured using the Rivermead Post-Concussion Questionnaire acutely and the extended Glasgow Outcome Score at three months.

Results/Conclusions The study included 81 patients (73 from CENTER-TBI, 8 local) with a median age of 44 years (range 14-85) and $57(70 \%)$ men. Within patients, cerebral white matter volume decreased (MR1/MR2 0.98, p=0.001) and ventricular volume increased (MR1/MR2 1.06, p<0.001). Compared to controls, white matter volume was normal on MR1 (patient/control 1.00, $\mathrm{p}=0.277$ ) but reduced on MR2 (patient/ control 0.97, $\mathrm{p}<0.001)$. Diffusion changes followed one of three trajectories: progressive injury, minimal change, or pseudonormalisation. Concussion symptoms worsened, improved and were variable in the three groups respectively (delta $[\mathrm{IQR}]+5.00[+2.00-+5.00],-4.5[-9.25-+1.75], 0.00[-6.25$ to +9.00$], p=0.018)$. MR1 predicted three-month outcome better than MR2 (AUC [95\% CI]: 0.93 [0.83-1.00] vs 0.72 [0.51-0.92]).

\section{THE FRAILTY IN MAJOR TRAUMA STUDY (FRAIL-T): FEASIBILITY OF NURSE LEAD FRAILTY ASSESSMENT IN ELDERLY TRAUMA AND THE IMPACT ON OUTCOMES}

${ }^{1}$ Heather Jarman, ${ }^{2}$ Robert Crouch, ${ }^{2}$ Mark Baxter, ${ }^{3}$ Bebhinn Dillane, ${ }^{4}$ Chao Wang, ${ }^{5}$ Elaine Cole. 'ED Clinical Research Unit, St George's Hospital; ${ }^{2}$ University Hospital Southampton NHS Foundation Trust; ${ }^{3} E D$ Clinical Research Unit.St George's Hospital; ${ }^{4}$ Faculty of Health, Social Care and Education, Kingston University and St George's; ${ }^{5}$ Blizard Institute, Queen Mary's, University of London

10.1136/emj-2020-rcemabstracts.30

Aims/Objectives/Background Frailty screening for major trauma patients has recently become part of the best practice commissioning tariff within NHS England, yet there is no consensus as to who should carry out this assessment or which tool best identifies frailty in the Emergency Department (ED). As the trauma population ages there is a need for accurate early identification of frailty in the ED to underpin frailty specific major trauma pathways. The primary aim of this study was to determine the feasibility and accuracy of ED nurse-led frailty assessment in patients $\geq 65$ years admitted to Major Trauma Centres (MTCs).

Methods/Design A prospective observational study was conducted across five UK MTCs, enrolling 370 participants over nine months. Eligible patients were aged 65 or more requiring trauma team activation. Frailty was assessed in the ED using three different tools: Trauma Specific Frailty Index (TSFI); Clinical Frailty Scale (CFS); PRISMA-7. ED nurse frailty assessment was correlated with Geriatrician assessment within 72 hours of admission using Spearman's correlation coefficient and kappa statistic for measuring the interrater agreement.

Results/Conclusions Complete frailty assessments were calculated for CFS in $99.4 \%$ of patients, PRISMA7 in $95.9 \%$ and TSFI in $37.58 \%$. Rates of frailty differed between tools: CFS 32\%, PRISMA7 57\% and TSFI 92\% whilst Geriatrician determined frailty was $37 \%$. In all tools frail patients were older $(\mathrm{p}<0.001)$ and falls $<2 \mathrm{~m}$ were the leading mechanism of injury $(p<0.05)$. CFS showed both strong correlation $\left(r_{s}\right.$ $0.639, \mathrm{p}<0.001$ ) and substantial agreement (kappa 0.637, $\mathrm{p}<0.001$ ) with Geriatrician assessment within 72 hours of admission.

ED nurses can accurately assess older major trauma patients for frailty using the Clinical Frailty Scale. These findings support assessment of frailty in the ED in order to identify patients who would benefit from early frailty specific care.

\section{SENIOR CLINICAL AND BUSINESS MANAGERS'PERSPECTIVES ON HOW DIFFERENT MECHANISMS AND MODELS OF EMPLOYING GENERAL PRACTITIONERS IN OR ALONGSIDE EMERGENCY DEPARTMENTS INFLUENCE WIDER SYSTEM OUTCOMES}

${ }^{1}$ Mazhar Choudrhey, ${ }^{1}$ Michelle Edwards, ${ }^{1}$ Alison Cooper, ${ }^{2}$ Pippa Anderson, ${ }^{3}$ Thomas Hughes, ${ }^{1}$ Andrew Carson-Stevens, ${ }^{2}$ Alison Porter, ${ }^{1}$ Edwards Adrian. ${ }^{1}$ Cardiff University; ${ }^{2}$ Swansea University; ${ }^{3}$ John Radcliffe Hospital

10.1136/emj-2020-rcemabstracts.31 